\title{
Methods for Scanning Transmission Electron Microscopy High Angle Annular Dark Field Based for Three Dimensional Analysis of the Local Composition in Solid Alloys
}

\author{
E. Rotunno ${ }^{1}$ V.Grillo ${ }^{2}$, T. Markurt ${ }^{3}$ and T. Remmele ${ }^{3}$ and M. Albrecht ${ }^{3}$
}

1. CNR-IMEM, Parco delle Scienze 37a, I-43100 Parma, Italy. (12pt)

2. CNR-Istituto Nanoscienze, Centro S3, Via G Campi 213/a, I-41125 Modena, Italy

3. Leibniz Institute for Crystal Growth, Max-Born-Strasse 2, 12489 Berlin, Germany

This paper reports on a novel approach to quantitatively reconstruct the number and the three-dimensional distribution of guest atoms inside a host matrix by a Scanning Transmission Electron Microscopy High Angle Annular Dark Field technique. Since the position of the guest atoms in the column strongly affects the chemical quantification for each column [1][2] this technique allows in addition for an improved quantification of the composition.

Our method is based on the joint analysis of a set of experimental data gained with variable beam convergence and/or defocus. It allows inverting the intensities into an atomic distribution along the columns for any dependence of the probe intensity on the thickness. It is therefore well suited to incorporate channeling effects that are usually neglected in other approaches [3]. As a case study we focus here on the systematic variation of the beam convergence that permits to set the maximum of the channeling oscillations at different depth [4]. We aim here to define the reliability and the limitation of this technique by the application of the method to accurate dynamic simulations in the case of the InGaN alloy. From Fig 1, showing the dependence of the probe intensity with depth inside an InGaN sample for convergence angles of 9, 15 and $21 \mathrm{mrad}$, it is evident that with changing the convergence angle we are probing different parts of the sample.

To extract detailed information we represent the guest atom distribution in a given column mathematically as a continuous profile and develop it in terms of harmonic components. The components can be determined from the experimental contrast at variable probe conditions by an inversion of the functional dependencies between these parameters.

To test the application of the method we simulated, with the multislice Frozen Phonon algorithm the images for the above mentioned convergences, which can realized in an aberration corrected STEM; on a supercell with a known guest atoms distribution. The results are shown in fig 2 a,b,c. Using the inversion algorithm we were able to retrieve the map of the number of atoms per column. In fig $2 \mathrm{~d}$,e the real composition per column and that retrieved by the algorithm are compared, showing a substantial agreement.

While the use of a single image permitted to obtain the quantification for a single colum with a confidence level less than $60 \%$, the new paradigm permits to obtain a confidence level of $95 \%$. . As a fringe benefit we are also able to retrieve a smooth, broad estimation of the guest atoms distribution along $\mathrm{z}$ that, for example, works very well for a single guest atom in the column. Fig $2 \mathrm{f}$ shows the superposition of the estimated distribution and the actual position. The agreement is quite good and 
remains satisfactory if the number of atoms in the cell is sufficiently low. We propose the method as a general framework to perform 3D quantitative analysis to be used in all multiple STEM-HAADF experiments including through-focal experiments.

[1] P.M. Voyles, J.L. Grazul, D.A. Muller Ultramicroscopy 96 (2003) 251-273

[2] E. Carlino V. Grillo Physical Review B 71 (2005) 235303.

[3] K.v. Benthem, A.R. Lupini, M. Kim, et al. Appl. Phys. Lett. 87, 034104 (2005)

[4] Y. Peng, P. D. Nellist and S. J. Pennycook Journal of Electron Microscopy 53 (2004) 257
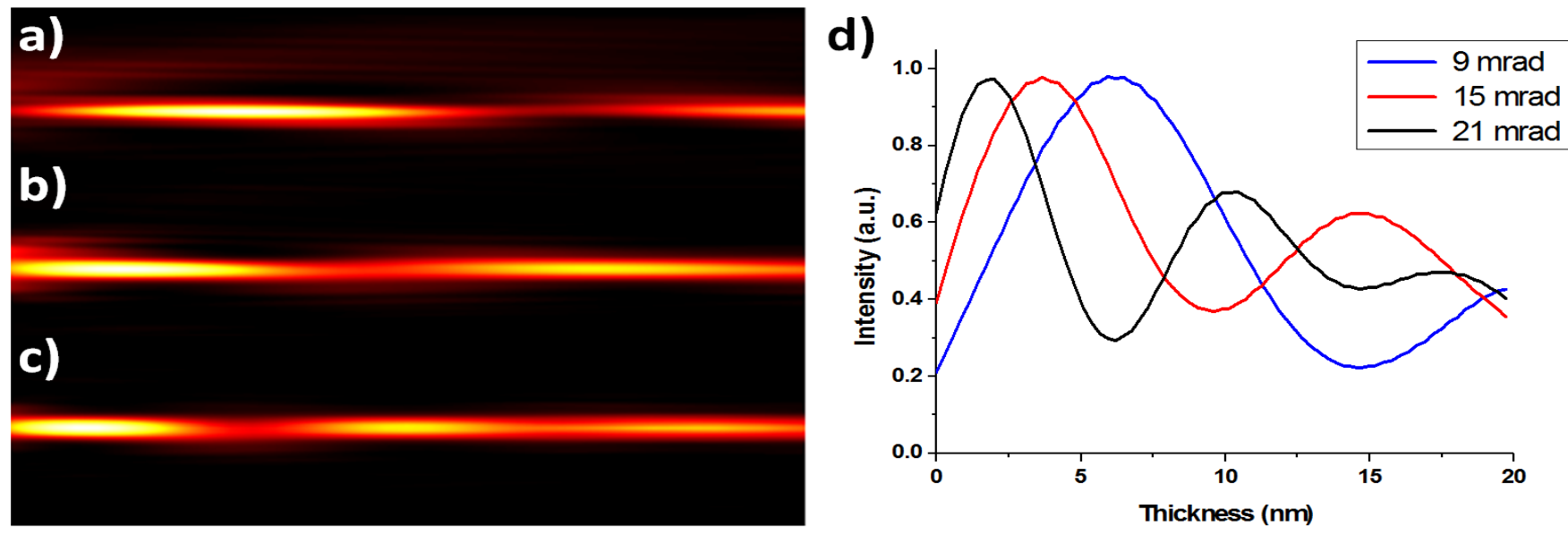

Figure 1. Probe intensity vs depth inside an InGaN sample for convergences of 9 (a), 15(b) and $21 \mathrm{mrad}$ (c). In fig $\mathrm{d}$ the profile in the center of the probe are plotted as a function of $\mathrm{z}$.

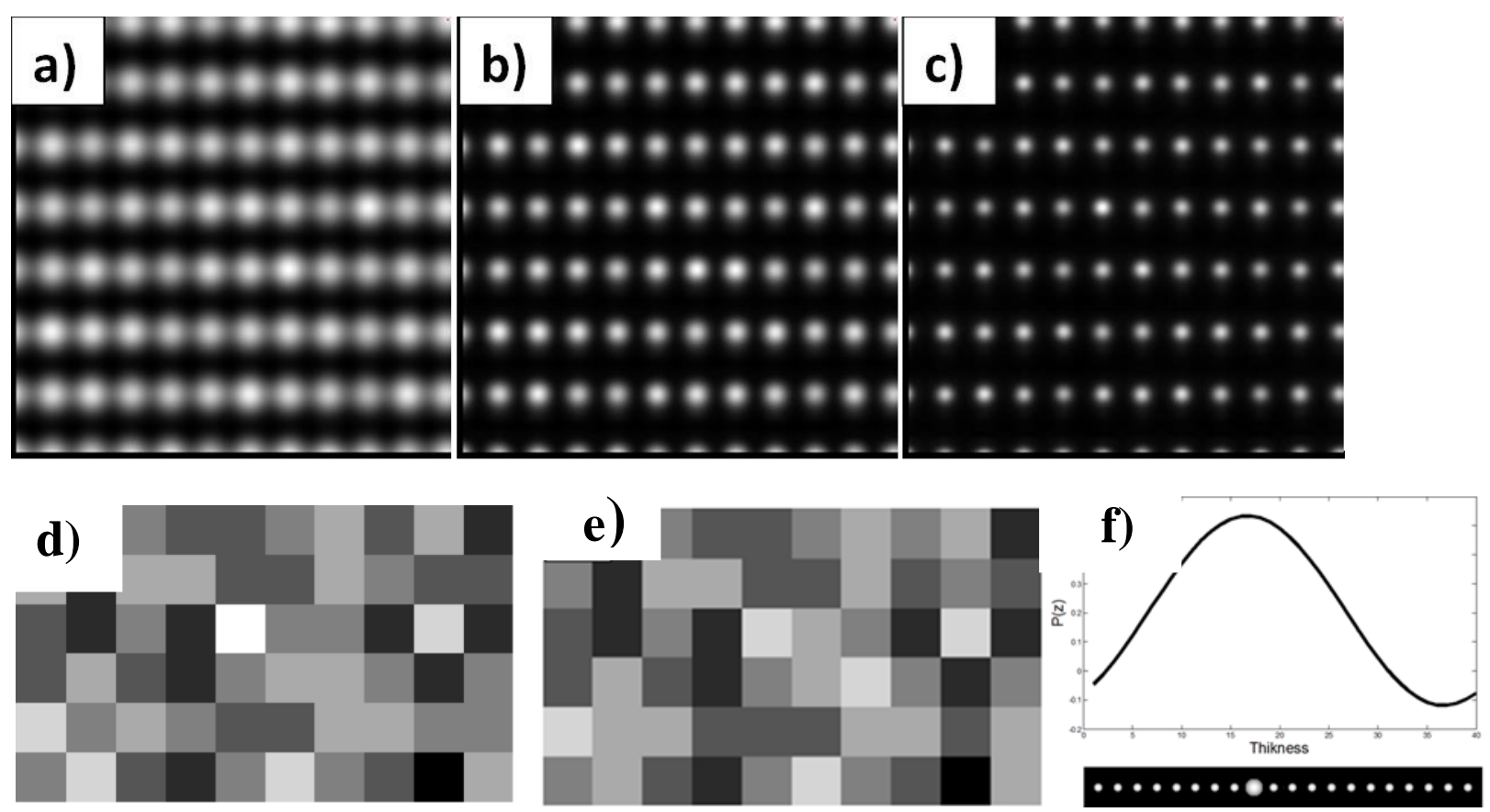

Figure 2. Simulation of a STEM-HAADF image of InGaN with convergence of of 9 (a), 15(b) and 21 mrad (c). Comparison of actual d) and retrieved e) column by column composition map. f) Retrieved "continuous" distribution of In atoms in a column compared with the actual distribution 